\title{
Determining the cause of stillbirth in Kumasi, Ghana
}

\author{
Jennifer N. Angell ${ }^{1}$ | Abdul-Razak S. Abdul-Mumin ${ }^{2}$ | Katherine J. Gold ${ }^{3,4, *}$
}

${ }^{1}$ Department of Anesthesiology and Perioperative Medicine, Oregon Health and Science University, Portland, OR, USA

${ }^{2}$ Department of Obstetrics and

Gynaecology, Komfo Anokye Teaching

Hospital, Kumasi, Ghana

${ }^{3}$ Department of Family Medicine, University of Michigan, Ann Arbor, MI, USA

${ }^{4}$ Department of Obstetrics and Gynecology, University of Michigan, Ann Arbor, MI, USA

\section{${ }^{*}$ Correspondence}

Katherine J. Gold, Department of Family Medicine, Department of Obstetrics and Gynecology, University of Michigan, Ann Arbor, MI, USA.

Email:ktgold@umich.edu

\section{Funding Information}

University of Michigan Global REACH

Office; University of Michigan Institute for Research on Women and Gender; University of Michigan Summer Biomedical Research Program; National Institutes of Health

\begin{abstract}
Objective: To classify cause-of-death (COD) for stillbirths occurring in a major referral hospital in Kumasi, Ghana.

Methods: In a retrospective review conducted between June 8, 2011, and June 12, 2012, detailed information was collected on all stillbirths delivered at Komfo Anokye Teaching Hospital in Kumasi, Ghana. Patient records were independently reviewed by investigators using the Perinatal Society of Australia and New Zealand's Perinatal Death Classification system to determine COD for each case.

Results: COD was analyzed in 465 stillbirth cases. The leading causes of death were hypoxic interpartum death $(105,22.6 \%)$, antepartum hemorrhage $(67,14.4 \%)$, hypertension (52, 11.2\%), and perinatal infection (32, 6.9\%). One hundred and fifty seven (33.8\%) stillbirths were classified as unexplained antepartum deaths.

Conclusions: This evaluation of stillbirth in a busy, tertiary care hospital in Kumasi, Ghana provides crucial insight into the high volume of stillbirth in Ghana as well as its medical causes. The study demonstrated the high rate of stillbirth attributed to hypoxic intrapartum events, placental abruption, pre-eclampsia, and unspecified bacterial infections. Yet, our rate of unexplained stillbirths underscores the need for a stillbirth classification system that thoughtfully integrates the needs and limitations of low-resource settings as unexplained stillbirth rates are a common indicator of the effectiveness of a classification system.
\end{abstract}

\section{KEYWORDS}

Cause of stillbirth; Factors associated with stillbirth; Ghana; Low-income countries; Obstetric complication; Stillbirth classification

\section{1 | INTRODUCTION}

Annually, 2.6 million fetuses are delivered stillborn across the globe. Ninety-eight percent of these are to mothers in low- and middleincome countries where poverty and lack of resources may limit access to basic obstetric care, medications, monitoring equipment, medical staff, emergency transportation, and facility-based delivery. ${ }^{1}$ The Global Burden of Disease Study 2015 found the highest rates of stillbirth to be in western and central sub-Saharan Africa with eight countries experiencing more than 25 stillbirths per 1000 pregnancies. ${ }^{2}$ Despite this alarming rate, research into stillbirth and the implementation of effective solutions has been particularly sparse in low-resource settings. ${ }^{3,4}$ There has been an international call to document fetal deaths through national statistics so that the scope of the problem can be adequately documented. ${ }^{4-6}$ Exact prevalence of stillbirth at 28 weeks and beyond in Ghana is unknown with estimates ranging from 14 to 40 per 1000 pregnancies. ${ }^{7-9}$ The Ghanaian government's 2017 Maternal Health Survey approximates the stillbirth rate at 40 per 1000 pregnancies. ${ }^{10}$ Furthermore, there is limited data on stillbirth cause-of-death (COD) in Ghana, which impedes the development of effective and economic interventions.

Multiple issues complicate the task of classifying stillbirth COD in a low-resource setting such as Ghana. These include limited early 
evaluation and documentation of risk factors, limited early dating to prevent post-date stillbirths, a limited availability of diagnostic testing, a large proportion of deliveries occurring outside of health facilities, and lack of early identification of fetal distress and prompt delivery of such fetuses. ${ }^{11}$ Absent or incomplete health records additionally complicate the ability to identify COD. Occasionally, non-medical family members who were present at the time of death or delivery are interviewed to help in determining COD, however, in Ghana, verbal autopsies for stillbirth have been shown to have poor concordance with hospital-assigned COD. ${ }^{12}$

The aim of the present study was to fill these gaps in knowledge through the collection of detailed data on third-trimester stillbirths at a tertiary hospital in Ghana, and by conducting a rigorous COD analysis to identify the most common contributors to fetal demise. These results could allow comparisons to other countries globally and could help guide future interventions to improve clinical outcomes.

\section{2 | MATERIALS AND METHODS}

From June 8, 2011, to June 12, 2012, we identified all stillbirths delivered at Komfo Anokye Teaching Hospital (KATH). KATH is in the center of the second largest city, Kumasi, and is one of the largest teaching hospitals in Ghana. Stillbirth was defined per WHO criteria as "a baby born with no signs of life at or after 28 weeks' gestation". ${ }^{13}$ The study was approved by the institutional review board at the University of Michigan and by the Committee on Human Research Publication and Ethics of the Kwame Nkrumah University of Science and Technology in Kumasi, which governs research at KATH. Consent was not required owing to the retrospective design, and data were de-identified.

The primary data abstractor (A-RSA-M) was a senior specialist obstetrician at KATH who visited the labor and delivery wards several times weekly to collect data from paper charts for stillbirth deliveries, to elicit missing data through discussion with on-duty midwives, and to assess staffing during the time of the patient's labor. A 92-point survey tool was constructed by our team to collect data required by various stillbirth classification systems and information on hospital staffing. For each fetal death we abstracted maternal demographics; past medical history; previous pregnancy outcomes; prenatal care (e.g. timing and number of prenatal care visits, malaria prophylaxis, use of ultrasound); prenatal test results (e.g. hemoglobin $(\mathrm{Hb})$, sickle cell, $\mathrm{Hb}$-electrophoresis, blood group, rhesus factor, VDRL, HIV, group B streptococcus); and admission and delivery information including complications and potential contributors to death. Placental pathology and fetal autopsy were almost never performed in this setting owing to lack of available resources. A second reviewer, an attending physician (KJG), and research staff re-abstracted data from hospital charts in Ghana for approximately $30 \%$ of cases to ensure accuracy, fill in missing data points, and confirm outlying data. Data were initially recorded on a written datasheet and then entered into a computer database (Access version 14; Microsoft, Redmond, WA, USA) and triple-checked for data entry accuracy.
In selecting a stillbirth classification system for a low-resource setting, we prioritized that the system: (1) be internationally applicable to allow global comparison of high-income countries with low- and middle-income countries; (2) be comprehensive in scope and categories while still accommodating gaps in documentation and limited access to diagnostic testing; (3) provide detailed category guidelines to facilitate inter-rater coding agreement; and (4) assign COD based on the initiating factor (e.g. placental abruption) that led to fetal demise rather than the final consequence (e.g. asphyxia). There are more than 80 stillbirth classification systems in existence. ${ }^{14}$ Upon reviewing the most prevalent systems, the Perinatal Society of Australia and New Zealand's Perinatal Death Classification (PSANZ-PDC) system was selected as most closely aligning with this study's priorities. ${ }^{15}$ Although it was designed for high-income countries, it was possible to use in the present study setting and has been shown to have a low proportion of unexplained stillbirths as well as satisfactory inter-rater agreement, information retention, and ease of coding. ${ }^{16}$

Certain elements of the PSANZ-PDC system had limited applicability in a low-resource setting as information on potential causes was typically restricted given the absence of test results or autopsy. We conferred with the original authors of the PSANZ-PDC to adapt the coding system for a low- or middle-income country and to clarify ambiguities in the coding guidelines to ensure we were appropriately selecting codes based on limited data. These modifications are detailed in Appendix S1: of particular interest are the flow charts describing the more nuanced forms of hypoxic intrapartum deaths.

One author of the present study (JNA), a medical student at the time of the study, created case summaries for each of the 465 documented stillbirths using the data collected. Two researchers (KJG and JNA) individually coded COD for each stillbirth based on the case summaries using the PSANZ-PDC system. Differences in coding assignment were then reviewed and discussed by the coders to determine a finalized COD. The initial agreement rate among reviewers was $77 \%$, with all disagreements resolved by consensus.

\section{3 | RESULTS}

Between June 2011 and June 2012, we identified 465 stillbirths at $\mathrm{KATH}$, at or after 28 weeks of gestation. The mean maternal age was 29, and 193 (41.5\%) women were married (Table 1). A total of 157 stillbirths (33.8\%) were classified as unexplained antepartum deaths. The most common identified COD by PSANZ-PDC category was hypoxic intrapartum death $(105,22.6 \%)$, primarily with no intrapartum complication $(27,25.7 \%)$ or cord prolapse $(18,17.1 \%)$. The second most frequent COD was antepartum hemorrhage (67, 14.4\%), primarily from placental abruption (61,91.0\%). Hemorrhage is followed by hypertension $(52,11.2 \%)$ and perinatal infection $(32,6.9 \%)$. The leading causes of death are exhibited in Figure 1 with "other" representing the less common categories: maternal conditions (22, $4.7 \%)$, congenital abnormality (20,4.3\%), and fetal growth restriction $(10,2.2 \%)$. The most common causes are further classified by subcategory in Table 2. 
TAB LE 1 Demographics of women $(n=465)$ who delivered stillborn fetuses. ${ }^{a}$

\begin{tabular}{|ll|}
\hline Characteristic & Value \\
\hline Age, $y$ & $29+6(14-46)$ \\
\hline$<20$ & $38(8.2)$ \\
\hline $20-29$ & $218(46.9)$ \\
\hline $30-39$ & $183(39.4)$ \\
\hline$>40$ & $26(5.6)$ \\
\hline Education & \\
\hline None & $65(14.0)$ \\
\hline Primary school & $166(35.7)$ \\
\hline Middle or junior secondary school & $160(34.5)$ \\
\hline Senior secondary school or higher & $38(8.2)$ \\
\hline Data missing & $36(7.7)$ \\
\hline Marital status & \\
\hline Married & $193(41.5)$ \\
\hline Living together & $93(20.0)$ \\
\hline Never married & $96(20.7)$ \\
\hline Widowed & $1(0.2)$ \\
\hline Data missing & $82(17.6)$ \\
\hline Gravida & $3.8 \pm 2.3$ \\
\hline Parity & $2.7 \pm 1.9$ \\
\hline Previous stillbirth & $44(9.5)$ \\
\hline
\end{tabular}

${ }^{a}$ Values are given as mean + SD (range) or number (percentage).

\section{4 | DISCUSSION}

To our knowledge, this is the first study to evaluate stillbirth COD in Ghana using a standardized, international classification system rooted in quantitative data. The only previous study of stillbirth COD in Ghana is an autopsy study completed in $1998 .{ }^{17}$ Most studies investigating stillbirth in Ghana have focused on maternal and neonatal risk factors such as young maternal age, poor prenatal care, prolonged labor, macrosomia, or low-birth weight, but rarely assign COD. ${ }^{18-20}$ Verbal autopsies have been conducted with Ghanaian community members to determine stillbirth COD in settings that lack reliable vital registration systems. ${ }^{21}$ However, evaluation of the verbal autopsy method in Ghana has shown a poor diagnostic accuracy rate of $64 \%$ compared to hospital records. ${ }^{12}$ Given the limitations of previous efforts to study stillbirth in Ghana, the present analysis provides crucial insight into the medical causes of death at a tertiary center.

The analysis demonstrates the high rate of stillbirth mortality associated with hypoxic intrapartum events, placental abruption, pre-eclampsia, and bacterial infections. A systematic review evaluating stillbirth in low- and middle-income countries found the rate of unexplained stillbirths to vary from $3.8 \%$ to $57.4 \%$; our finding that a third of stillbirths in the present study were unexplained is consistent with previous studies. ${ }^{22}$ The unexplained stillbirth rates were highest in low-income countries included in the review, such as: Bangladesh

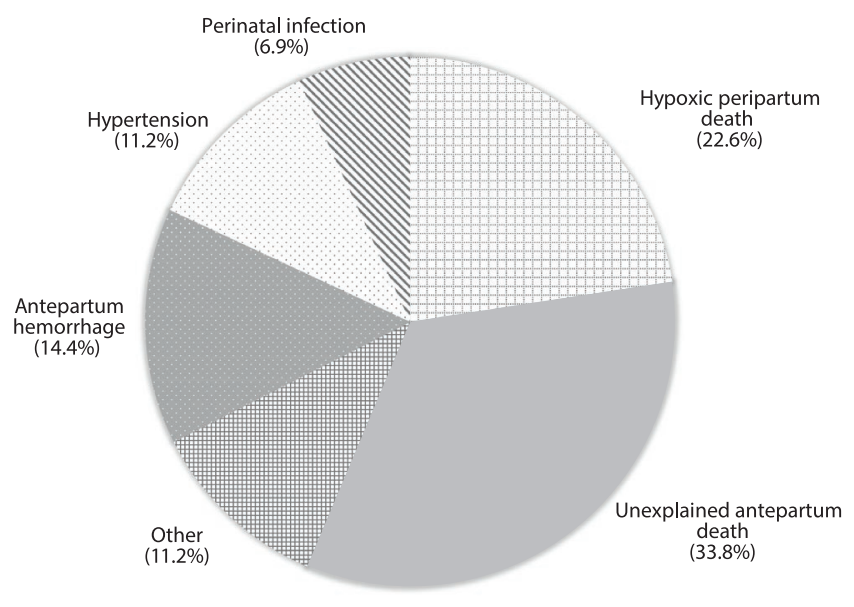

FIGURE 1 Leading cause-of-death by percentage.

(49\%), Nepal (47\%), Nigeria (38.8\%), and India (18\%). ${ }^{22}$ This broad range of unexplained stillbirths reflects the variability of classification systems in coding guidelines and requirements. A comparative study of contemporary stillbirth classification systems implemented six systems primarily in developed countries. ${ }^{16}$ The unexplained stillbirth rate differed distinctly by system: CODAC 9.5\%, Tulip 10.2\%, ReCoDe 13.8\%, PSANZ-PDC 15.4\%, Aberdeen 44.3\%, Wigglesworth 50.2\%. ${ }^{16}$ Our unexplained rate underscores the need for a stillbirth classification system that thoughtfully integrates the needs and limitations of low-resource settings as unexplained stillbirth rates are a common indicator of the effectiveness of a classification system.

A recent Lancet report describes the rate of stillbirths attributed to hypertension, pre-eclampsia, and eclampsia as $15 \%$ in developing countries, ${ }^{6}$ which is similar to the $11 \%$ noted in the present study. The systematic review of stillbirth in developing countries reported a similar rate of hypoxic COD compared with the present study findings (23\% vs $25 \%$ ) but a much higher rate of infectious deaths $(7 \%$ vs $50 \%)^{21,22}$ Patient antenatal record books and medical records at KATH and other referring facilities often lacked prenatal testing data and rarely documented maternal or fetal laboratory testing after stillbirth (if performed) which limited our ability to identify infections that may have contributed to death.

Hypoxic intrapartum events were the second-leading cause of stillbirth at KATH. In contrast to high-resource countries where intrapartum stillbirths are rare, hypoxic events are far more common and thought to be potentially preventable with improved fetal monitoring, better access, staffing, equipment, and medication for assisted and cesarean delivery. Hypertensive causes of death were common in this study suggesting that increasing the number of late-pregnancy prenatal visits and testing for women with elevated blood pressure, as well as expanding community education about pre-eclampsia symptoms, may help to ultimately reduce the associated risk of stillbirth by identifying women at risk and encouraging rapid care. Intrapartum blood pressure monitoring and treatment of severe hypertension may also improve fetal outcomes and reduce rates of acute abruption. Of note, eight (18\%) of the stillbirths assigned pre-eclampsia as a COD were, in fact, secondary to eclampsia. The PSANZ-PDC system does not have a 
TABLE 2 Breakdown of hypoxic, hemorrhagic, hypertensive, infectious, and maternal-related deaths.

\begin{tabular}{|c|c|}
\hline Category & No. $(\%)^{a}$ \\
\hline \multicolumn{2}{|l|}{ Hypoxic intrapartum death $(n=105)$} \\
\hline $\begin{array}{l}\text { No intrapartum complications and no evidence of } \\
\text { NRFS }^{\text {b }}\end{array}$ & $27(25.7)$ \\
\hline Cord prolapse & $18(17.1)$ \\
\hline Other & $17(16.2)$ \\
\hline \multicolumn{2}{|l|}{ Cephalopelvic disproportion } \\
\hline \multicolumn{2}{|l|}{ Macrosomia } \\
\hline \multicolumn{2}{|l|}{ Footling breech } \\
\hline \multicolumn{2}{|l|}{ Retained second twin } \\
\hline \multicolumn{2}{|l|}{ Transverse lie } \\
\hline \multicolumn{2}{|l|}{ Preterm rupture of membranes } \\
\hline \multicolumn{2}{|l|}{ Premature preterm rupture of membranes } \\
\hline \multicolumn{2}{|l|}{ Preterm labor } \\
\hline Unspecified hypoxic intrapartum death & $17(16.2)$ \\
\hline Uterine rupture & $12(11.4)$ \\
\hline Evidence of NRFS in a normally grown infant & $10(9.5)$ \\
\hline Shoulder dystocia & $2(1.9)$ \\
\hline Intrapartum complications & $2(1.9)$ \\
\hline \multicolumn{2}{|l|}{ Antepartum hemorrhage $(n=67)$} \\
\hline Placental abruption & $61(91)$ \\
\hline Placenta previa & $6(8.9)$ \\
\hline \multicolumn{2}{|l|}{ Hypertension ( $n=52)$} \\
\hline Pre-eclampsia & $37(71.2)$ \\
\hline Pre-eclampsia superimposed on chronic hypertension & $8(15.4)$ \\
\hline Chronic hypertension & $3(5.8)$ \\
\hline Gestational hypertension & $2(3.8)$ \\
\hline Unspecified hypertension & $2(3.8)$ \\
\hline \multicolumn{2}{|l|}{ Perinatal infection $(n=32)$} \\
\hline Unspecified bacterial & $12(37.5)$ \\
\hline Other viral (e.g. HIV) & $8(25.0)$ \\
\hline Spirochetal (e.g. syphilis) & $5(15.6)$ \\
\hline Protozoal (e.g. toxoplasma) & $5(15.6)$ \\
\hline Other bacterial (e.g. tuberculosis) & $1(3.1)$ \\
\hline Other unspecified organism & $1(3.1)$ \\
\hline \multicolumn{2}{|l|}{ Maternal conditions $(n=22)$} \\
\hline Other specified maternal conditions & $16(72.7)$ \\
\hline \multicolumn{2}{|l|}{ Sickle cell anemia } \\
\hline \multicolumn{2}{|l|}{ Severe anemia (i.e. hemoglobin $<7.0 \mathrm{~g} / \mathrm{dL}$ ) } \\
\hline \multicolumn{2}{|l|}{ HIV } \\
\hline Diabetes/gestational diabetes & $4(18.2)$ \\
\hline Accidental injury & $1(4.5)$ \\
\hline Maternal sepsis & $1(4.5)$ \\
\hline
\end{tabular}

${ }^{\mathrm{a}}$ Total percentages may equal more than $100 \%$ due to rounding.

${ }^{\mathrm{b}}$ Non-reassuring fetal status. sub-classification of eclampsia, and discussion with one of its authors led us to classify with pre-eclampsia instead.

Some adaptations were required to fully utilize the PSANZ-PDC system in this resource-limited setting. For example, PSANZ-PDC demands rigorous criteria to classify a death as caused by perinatal infection. It requires histological confirmation of infection in cord/placenta or fetus, or convincing clinical evidence of primary maternal infection and positive culture of a pathogen from mother or placenta. While screening for HIV, hepatitis B, and syphilis were routine at KATH, additional cultures were very rarely available. Thus, we agreed that clinical evidence suggestive of infection affecting the fetus (e.g. foul-smelling amniotic fluid) would be sufficient to code as perinatal infection in the absence of evidence pointing toward other causes of death. This decision was motivated by results of the one other study to utilize PSANZ-PDC in a low-resource setting. In that instance, zero deaths were attributed to perinatal infection, likely due to the rigorous classification criteria rather than an actual lack of perinatal infection. ${ }^{23}$ Although PSANZ-PDC provides a classification guide for coders, for several items we asked for clarification from the system's authors in order to facilitate practical use of the classification in our setting. These clarifications are provided in the appendix for transparency and may be useful to aid other users implementing PSANZ-PDC in similar settings.

These coding adaptations were essential in order to be able to use PSANZ-PDC in the present study setting, but could be a potential limitation in comparing our results to other studies. The research is additionally limited by retrospective data collection, a lack of rigorous documentation inherent in the paper medical charts from which we sourced most data, and the difficulty in obtaining laboratory tests and placental pathology in a country with very limited resources. Also, the data may not be generalizable to regions of Ghana outside Kumasi, as KATH is a tertiary referral hospital and sees the highest-risk patients in the region.

The process of scientifically assigning stillbirth COD is convoluted by the lack of a single internationally-accepted classification system. In the authors' evaluation of the numerous available classification systems, less desirable systems were the Wigglesworth and Aberdeen, which were both found to have poor inter-rater agreement and a high proportion of unexplained stillbirths. ${ }^{16}$ The WHO ICD-Perinatal Mortality classification system was reviewed by our team as well and determined to be less suitable, as the COD classification is based primarily upon the clinician's clinical decision-making with no guidelines to assist in systematically assigning the main underlying etiology. The Global Network Classification System was initially intriguing as it was designed with the low-resource setting in mind, but we were concerned it may be too minimalistic for the data we had available, resulting in less specific causes than we could identify, and it assigned COD based on the final event rather than the initiating factor. An attractive feature of the PSANZ-PDC system is the assignation of COD based on the initiating factor in the chain of events, but this too can be limiting as there may be cases in which the final event could be more causative than the initiating.

The international stillbirth research community has been seeking a system that could standardize stillbirth COD classification to one effective, thorough system that could be applied across settings despite 
resource limitations. ${ }^{14,16}$ The PSANZ Perinatal Death Classification system was updated in March 2018, and a working group through the International Stillbirth Alliance is developing a new, standardized stillbirth classification system which will likely have some similarity to PSANZ-PDC. ${ }^{24}$

The present investigation provided critical insights into the high volume of stillbirths in Ghana which is generalizable to similar hospitals in sub-Saharan Africa. The study shone a light on stillbirth in Ghana and provided data to support the future development of clinical and policy-driven interventions. It demonstrated both the strengths and weaknesses of using the PSANZ-PDC system in a low-income country, as has only been completed once before, and we hope this analysis will assist with further refining an ideal COD classification system.

\section{AUTHOR CONTRIBUTIONS}

JNA contributed to background literature review, data analysis, and manuscript preparation. A-RSA-M contributed to the design of the study, data acquisition, and revising the manuscript. KJG contributed to the conception and design of the study, background literature review, data acquisition and analysis, and manuscript preparation.

\section{ACKNOWLEDGMENTS}

Funding for travel was provided by grants from the University of Michigan Global REACH Office and the University of Michigan Institute for Research on Women and Gender. J.N.A. received salary support from the University of Michigan Summer Biomedical Research Program. K.J.G. received salary support from the National Institutes of Health as part of a K-23 training grant. No funder had a role in analysis of results or preparation/review of the manuscript. We thank Dr. Vicki Flenady for sharing her expertise on the PSANZ Perinatal Death Classification system and assisting our team with interpreting the system's coding guidelines for the low-resource setting. We are grateful to the midwives at KATH for providing helpful data about staffing and census at the time of each stillbirth. Additionally, we express deep gratitude to our colleague, Martha Boggs, for her meticulous assistance with data review.

\section{CONFLICTS OF INTEREST}

The authors have no financial conflicts of interest.

\section{REFERENCES}

1. Mullan Z, Horton R. Bringing stillbirths out of the shadows. Lancet. 2011;377:1291-1292.

2. GBD 2015 Child Mortality Collaborators, Bhutta ZA, Coates MM, et al. Global, regional, national, and selected subnational levels of stillbirths, neonatal, infant, and under-5 mortality, 1980-2015: A systematic analysis for the Global Burden of Disease Study 2015. Lancet. 2016;388:1725-1774.

3. Lawn JE, Gravett MG, Nunes TM, Rubens CE, Stanton C. Global report on preterm birth and stillbirth (1 of 7): Definitions, description of the burden and opportunities to improve data. BMC Pregnancy Childbirth. 2010;10(Suppl 1):S1.

4. McClure EM, Saleem S, Pasha O, Goldenberg RL. Stillbirth in developing countries: A review of causes, risk factors and prevention strategies. J Matern Neonatal Med. 2009;22:183-190.

5. Lawn JE, Yakoob M, Haws RA, Soomro T, Darmstadt GL, Bhutta ZA. 3.2 million stillbirths: Epidemiology and overview of the evidence review. BMC Pregnancy Childbirth. 2009;9(Suppl 1):S2.

6. Lawn JE, Blencowe H, Waiswa P, et al. Stillbirths: Rates, risk factors, and acceleration towards 2030. Lancet. 2016;387: 587-603.

7. Ghana Statistical Service. Ghana Demographic Health Survey[Website]. https://dhsprogram.com/pubs/pdf/FR307/FR307. pdf. Accessed March 02, 2018.

8. Engmann C, Walega P, Aborigo RA, et al. Stillbirths and early neonatal mortality in rural Northern Ghana. Trop Med Int Heal. 2012;17:272-282.

9. Edmond KM, Quigley MA, Zandoh C, et al. Aetiology of stillbirths and neonatal deaths in rural Ghana: Implications for health programming in developing countries. Paediatr Perinat Epidemiol. 2008;22:430-437.

10. Ghana Statistical Service (GSS), Ghana Health Service (GHS) and ICS. Ghana Maternal Health Survey 2017: Key Indicators Report. Accra, Ghana: ; 2018.

11. Moyer CA, McLaren ZM, Adanu RM, Lantz PM. Understanding the relationship between access to care and facility-based delivery through analysis of the 2008 Ghana Demographic Health Survey. Int J Gynecol Obstet. 2013;122:224-229.

12. Edmond KM, Quigley MA, Zandoh C, et al. Diagnostic accuracy of verbal autopsies in ascertaining the causes of stillbirths and neonatal deaths in rural Ghana. Paediatr Perinat Epidemiol. 2008;22: 417-429.

13. World Health Organization. Stillbirths [WHO website]. 2016. http:// www.who.int/maternal_child_adolescent/epidemiology/stillbirth/ en/. Accessed June 14, 2018.

14. Leisher SH, Teoh Z, Reinebrant H, et al. Classification systems for causes of stillbirth and neonatal death, 2009-2014: An assessment of alignment with characteristics for an effective global system. BMC Pregnancy Childbirth. 2016;16:269.

15. Perinatal Society of Australia and New Zealand. Perinatal Mortality Classifications [Website]. 2009. http://www.stillbirthalliance.org. au/doc/Section_7_Version_2.2_April_2009.pdf. Accessed August 6, 2018.

16. Flenady V, Frøen JF, Pinar H, et al. An evaluation of classification systems for stillbirth. BMC Pregnancy Childbirth. 2009;9:1-13.

17. Wiredu EK, Tettey Y. Autopsy studies on still births in Korle Bu Teaching Hospital. II: Causes of death in 93 still births. West Afr J Med. 1998;17:148-152.

18. Badimsuguru $A B$, Nyarko KM, Afari EA, Sackey SO, Kubio C. Determinants of stillbirths in Northern Ghana: A case control study. Pan Afr Med J. 2016;25(Supp 1):18.

19. Afulani PA. Determinants of stillbirths in Ghana: Does quality of antenatal care matter? BMC Pregnancy Childbirth. 2016;16:1-17.

20. Agbozo F, Abubakari A, Der J, Jahn A. Prevalence of low birth weight, macrosomia and stillbirth and their relationship to associated maternal risk factors in Hohoe Municipality, Ghana. Midwifery. 2016;40:200-206

21. Manortey S, Carey A, Ansong D, et al. Verbal autopsy: An analysis of the common causes of childhood death in the Barekese sub-district of Ghana. J Public Health Afr. 2011;2:e18.

22. Aminu M, Unkels R, Mdegela M, et al. Causes of and factors associated with stillbirth in low- and middle-income countries: A systematic literature review. BJOG. 2014;121:141-153.

23. Hirst JE, Ha LTT, Jeffery HE. Reducing the proportion of stillborn babies classified as unexplained in Vietnam by application 
of the PSANZ clinical practice guideline. Aust N Z J Obstet Gynecol. 2012;52:62-66.

24. Perinatal Society of Australia and New Zealand. Clinical Practice Guideline for Care Around Stillbirth and Neonatal Death Section 7 The PSANZ Classification System for Stillbirths and Neonatal Deaths [Website]. 2018. https://sanda.psanz.com.au/assets/Uploads/Secti on-7-PSANZ-Classification-of-perinatal-deaths-V3-23032018.pdf. Accessed August 7, 2018.

\section{SUPPORTING INFORMATION}

Additional supporting information may be found online in the Supporting Information section at the end of the article.

Appendix S1. Adaptation of PSANZ-PDC for Use in a LowResource Country. 